\title{
Thermography of Asteroid Ryugu by Hayabusa2
}

\author{
by T. Okada* and Hayabusa2 TIR Team*
}

* Institute of Space and Astronautical Science, Japan Aerospace Exploration Agency, 3-1-1 Yoshinodai, Chuo, Sagamihara, 252-5210 Japan, okada@planeta.sci.isas.jaxa.jp

\section{Abstract}

Thermography of the C-type Near Earth Asteroid 162173 Ryugu has revealed the thermophysical properties of the surface of the primitive solar system small body. Thermal Infrared Imager TIR is a remote sensing instrument onboard Hayabusa2, the Japanese second asteroid sample return mission. TIR is based on two-dimensional uncooled microbolometer array with $328 \times 248$ effective pixels, $16.7^{\circ} \times 12.7^{\circ}$ field of view, and a single band of 8 to $12 \mu \mathrm{m}$ wavelength range. New results of global, local and close-up thermal images of the asteroid are briefly reported.

\section{Introduction}

Global, local and close-up thermal images of the C-type Near-Earth asteroid 162173 Ryugu were taken by the Thermal Infrared Imager TIR [1] on Hayabusa2 to investigate its thermophysical properties. TIR is a two-dimensional thermographic camera developed to study the nature of Ryugu and its origin and evolution. The instrument is also used for safe landing for sample collection regarding the assessment for the surface thermal environment and the hazardous boulder abundance. Demonstration to utilize thermographic camera in planetary missions is another purpose. Outlines of the TIR observations during the asteroid rendezvous phase are briefly described.

\section{Hayabusa2 and the target asteroid Ryugu}

Hayabusa2 [2] is an asteroid mission to explore the C-type asteroid, after the Hayabusa mission [3] which visited and returned sample from S-type near-earth asteroid 25143 Itokawa. Hayabusa2 arrived at Ryugu on 27 June 2018 and started remote sensing including TIR to characterize the asteroid regarding its shape, spin state, geomorphology, spectral and thermal properties, geologic features, and gravity. Such information is used for understanding the nature of the asteroid and for proper landing site selection for the sample collection and the release of landers. Descent operations have been conducted, during which close-up imaging was carried out by TIR. One of them is to release and track a Target Marker (TM), which has proven that TIR can detect the $10-\mathrm{cm}$ sized TM on the asteroid surface. The first sampling of the asteroid surface material has taken place on 22 February 2019, where close-up thermal images nearby the landing site have been taken just before the touchdown. The impact experiment using the Small Carry-on Impactor (SCI) has taken place to expose the subsurface materials on 5 April 2019 to collect the ejected materials around the impact crater. Hayabusa2 will return those samples to Earth in 2020.

Ground-based observations [4] predicted that Ryugu is a C-type near-earth asteroid, of roughly rounded shape with a diameter of about $0.9 \mathrm{~km}$. It rotates in 7.63 hours. Its geometric albedo is about 0.05 or lower, and the globally averaged thermal inertia is 150 to 300 [tiu $=\mathrm{J} \mathrm{m}^{-2} \mathrm{~s}^{-0.5} \mathrm{~K}^{-1}$ ], predicting the surface covered with coarse regolith about $\mathrm{cm}$ sized granules. These predictions are basically consistent with the on-site observations, but the shape is top-shaped, and the surface is not covered with regolith like granules but larger rocks. No flat and smooth area larger than $10 \mathrm{~m}$ scale was found on the surface of Ryugu for easy and safe landing of the Hayabusa2 spacecraft [5].
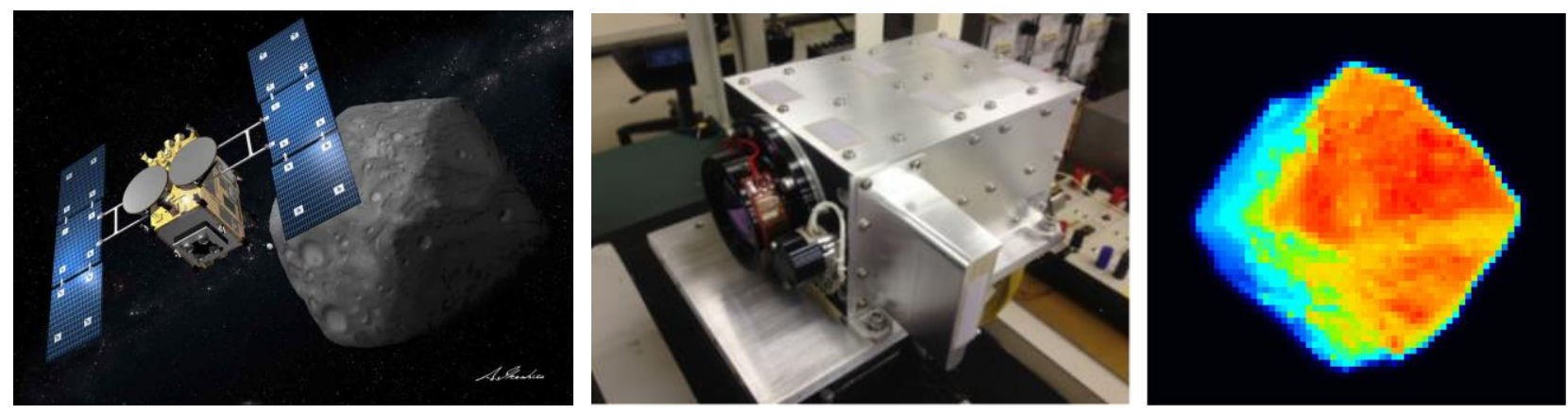

Fig. 1: An artist illustration of Hayabusa2 mission (left, JAXA/lkeshita), an image of the thermal infrared imager (TIR) on Hayabusa2 (middle), and a thermal image of asteroid Ryugu at dusk side by TIR on 25 Aug 2018 (right). 


\section{TIR Observations in Proximity Phase}

\subsection{Global Thermal Images of Ryugu}

Thermal images of Ryugu have been taken from the Home Position (HP), $20 \mathrm{~km}$ from the asteroid. The surface temperature (mainly sunlit side, SPE angle $<20^{\circ}$ ) has been measured at 300 to $370 \mathrm{~K}$ at about 1 au from the Sun, and the temperature is gradually decreased inversely as the solar distance increases. Comparison with numerical thermal modeling shows that the surface of Ryugu is colder than expected in the case of fine regolith surface (thermal inertia $(\mathrm{TI})=50$ tiu) but hotter than thought in the case of base rocks ( $\mathrm{TI}>1000 \mathrm{tiu}$ ). It was interesting that most of large boulders with the size of several tens of meters on Ryugu show temperatures almost the same as the surrounding surfaces.

It was also found that the diurnal temperature profiles of Ryugu are rather flatter than expected for the flat surface covered with sandy layers like the Moon. The preliminary derived thermal inertia is most consistent from 200 to 500 tiu. This implies that the surface physical state of Ryugu is consistent with rough and porous surface where boulders might have high porosity (30 to $50 \%$ ) and the rest of the surrounding surface is dominated by porous rocks larger than the thermal skin depth of a few centimeters. It is important that such prediction of the surface physical state from the global thermal images are confirmed later by the close-up thermal imaging and detailed interpretation.

\subsection{High Resolved Local Thermal Images of Ryugu}

Higher resolved global to local thermal images of Ryugu were taken by TIR from $5 \mathrm{~km}$ altitude, and on the way to the lower altitude for the touchdowns or the release of landers. Those data will be used as the reference temperatures for thermal calculation of the spacecraft. In those images, geologic features such as the surface boulders are clearly distinguished from the surroundings. The temperatures of boulders and the diurnal temperature profiles show basically the same profiles as those at HP.

\subsection{Close-up Thermal Images of Ryugu}

Close-up thermal images of local sites of Ryugu have been taken by TIR at altitudes below $100 \mathrm{~m}$. This is the very unique and the first data set in planetary missions. Close-up thermal images show the surface physical state and a variety of boulders. As seen on optical images and the surface landers Minerva-II and Mascot, the surface of Ryugu is widely covered with centimeter to meter sized rocks and boulders, but not with fine regolith. Most of boulders in the close-up thermal images by TIR also show similar temperatures to the surroundings. There are limited number of boulders that show "Cold Spots", remarkably colder by 20 or $30 \mathrm{~K}$ than the surroundings, indicating dense rocks with lower porosity, indicating the thermal inertia of the volatile-rich carbonaceous chondrite meteorites of 700 to 1000 tiu.

\section{Technical Usage of Thermal Images}

Thermal images by TIR will be used for other purposes. The Target Marker (TM) is a spherical ball wrapped with recursively reflection sheet and was clearly imaged after its release and settlement on the asteroid surface. TM was identified by a TIR thermal image during its descent from the spacecraft and tracked with TIR images every 32 second before its settlement beside a large boulder. It is a good length scale on the asteroid. The SCI (Small Carry-on Impactor) was tracked by TIR images with the background against the asteroid surface, taken every 2 seconds after its release. The position and the attitude of $\mathrm{SCl}$ relative to the asteroid surface have been investigated using the TIR thermal images. The movement and nutation of the $\mathrm{SCl}$ is traced relative to the asteroid surface. These are the advantages of thermal images compared to optical images, because the target material and the asteroid surface as the background can be simultaneously imaged. This advantage will be utilized in the future lunar and planetary surface exploration to image the artificial and natural materials at the same time, or image the sunlit and shadowed surfaces simultaneously.

\section{REFERENCES}

[1] Okada T, Fukuhara T, Tanaka S, et al. Thermal Infrared Imaging Experiments of C-Type Asteroid 162173 Ryugu on Hayabusa2, Space Science Reviews, 2017; 208: 255-286. doi: 10.1007/s11214-016-0286-8.

[2] Tsuda Y, Nakazawa S, Kushiki K, et al. Flight status of robotic asteroid sample return mission Hayabusa2, Acta Astronautical 2016;127:702-709.

[3] Fujiwara A, Kawaguchi J, Yeomans D.K. et al. The Rubble-Pile Asteroid Itokawa as Observed by Hayabusa. Science 2006;312:1330-1334.

[4] Mueller TG, Durech J, Ishiguro M et al. Hayabusa-2 mission target asteroid 162173 Ryugu (1999 JU3): Searching for the object's spin-axis orientation, A\&A 2017;599:A103.

[5] Watanabe S, Hirabayashi M, Hirata N et al. Hayabusa2 arrives at the carbonaceous asteroid 162173 Ryugu - A spinning top-shaped rubble pile, Science 2019;364:268-272. doi: 10.1126/science.aav8032. 